\title{
The Evolution and Prospect of Research on Civil Servants' Job Burnout in China-Bibliometric Research Based on Document Journals
}

\author{
Zupeng Yang ${ }^{1, *}$ and Yuan $\mathrm{Su}^{2}$ \\ ${ }^{1}$ School of Government Management, Beijing Normal University, Haidian District, Beijing 100875, China \\ ${ }^{2}$ School of Education, Minzu University of China, Haidian District, Beijing 100081, China \\ *Corresponding author. Email:945412642@qq.com
}

\begin{abstract}
In order to understand the research hotspots and progress of civil servants' job burnout in China, the paper takes 163 documents published by CNKI from 2001 to 2020 as samples. The paper takes Citespace software as the analysis tool and assists Excel software to visually analyze the samples' time, authors, institutions, keywords, and causes. The purpose of this paper is to explore the causes and related problems of China's civil servants' job burnout, which can play a certain reference significance for domestic scholars to study civil servants' job burnout to improve China's civil service system and administrative performance and serve the people better.
\end{abstract}

Keywords: Civil servants; Job burnout; CNKI; Citespace

\section{INTRODUCTION}

Occupational burnout is a social phenomenon. As a special occupational area, the civil service system should arouse our attention and thinking. As managers who exercise state power and perform state administrative functions in accordance with law, civil servants' job burnout is more harmful. Since the 18th National Congress of the Communist Party of China, under the new political normal, the civil servants, especially the grassroots civil servants, have complained about "it is not easy to be an official", "it's hard for an official to make a living" and the mediocre government of "being an official but doing nothing" job burnout phenomena such as sloth administration. The job burnout of civil servants has aroused widespread social discussion. This paper uses bibliometric methods to study all the 163 journal papers on the job burnout of officials and civil servants collected by CNKI, and uses the knowledge network analysis tool---Citespace, combined with Excel, draws a knowledge map of domestic job burnout from the aspects of spatio-temporal distribution, keyword clustering, and cause analysis. We put forward our analysis in order to improve the job burnout of civil servants.

\section{DATA SOURCES AND RESEARCH METHODS}

\subsection{Document Source and Processing}

This research uses CNKI as the data retrieval source and collects data through the "Advanced Search" module in the "Journal Search". The search formula is: subject heading = "official burnout" or "cadre burnout" or "civil servant burnout" or "assigned graduates burnout"; Time span = 2001-2020; Journal source $=$ all journals; Search condition $=$ accurate. A total of 195 related documents were obtained (retrieval time is January 10, 2020). At the same time, in order to ensure the accuracy of the research, 22 papers for university management cadres and student cadres, 2 for nurses, 2 for hospital cadres and health care workers, and 1 for cadet cadres were eliminated. Duplicate records were removed, and 163 valid journal papers were finally screened out.

\subsection{Analysis Methods and Tools}

The bibliometric method is a research method that uses academic documents as the research object, analyzes the law of the documents from a quantitative perspective, and shows the research hotspots and development trends of the subject. Citespace software is an information visualization software developed by Professor Chaomei Chen in 2004 based on co-citation analysis and pathfinder network scaling (PFNET), using the Java computer programming language. 
Through the quantitative analysis of documents in specific research fields, the main content of domestic civil servants' job burnout research is visualized by using the functions of CiteSpace software such as keyword analysis, keyword clustering, hot word emergence analysis, and hot word spatio-temporal distribution. At the same time, using Excel software as a supplement. This paper makes an overall description for the external characteristics of domestic civil servants' job burnout, such as the annual documents distribution, core author distribution, author's institution distribution, and journal distribution.

\section{TIME AND SPACE ANALYSIS OF CIVIL SERVANT BURNOUT RESEARCH}

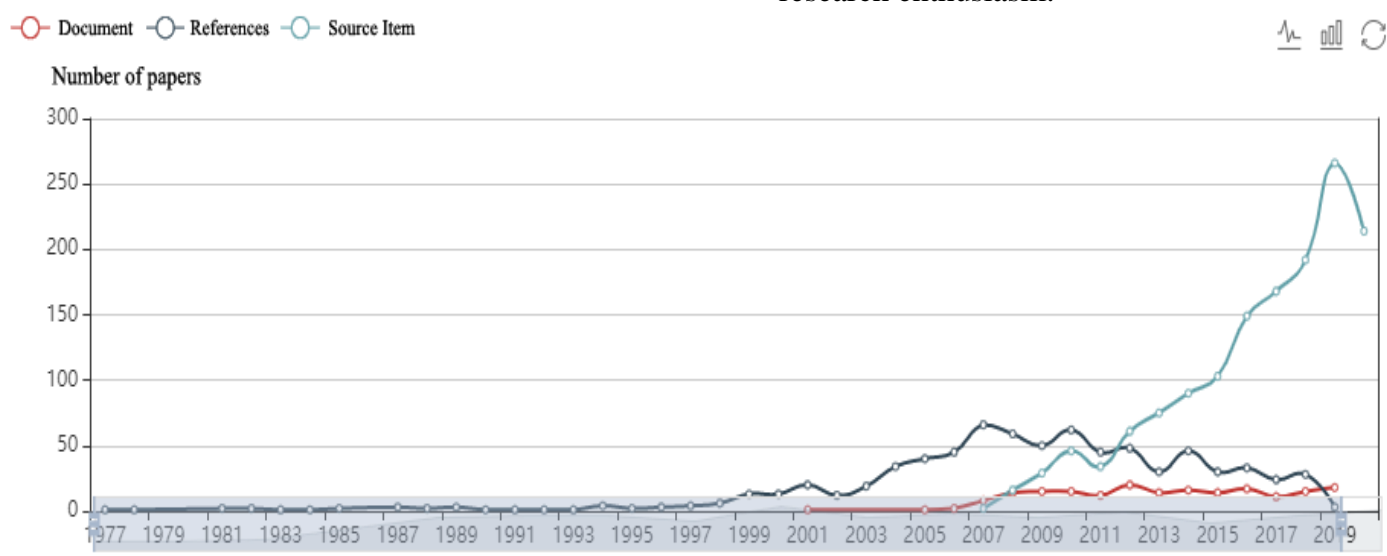

Figure 1. Number of publications on official burnout research

\subsection{Research Spatial Distribution}

\subsubsection{Author Analysis}

Table 1 is the analysis of the number of papers published by the authors. Judging from the number of papers published by the authors, only one author has published three papers on the theme of "official burnout". Xingrong Bao analyzed

\subsection{Research Time Distribution}

The number of annual publications is an important indicator to measure the evolution and development trend of the research on civil servants' job burnout. Figure 1 shows the publishing time chart, in which blue lines, green lines, and orange lines respectively indicate the publication time trends of selected documents, references, and certified documents. From 2001 to 2019, 163 research papers on official burnout were published in China, and the total number of publications was relatively small, indicating that the attention and degree of research on civil servant burnout are obviously insufficient. In 2009, 2012, 2014, and 2016, the number of publications was the largest, with $15,18,15$, and 17 respectively, indicating that the research on civil servant burnout is fluctuating and has not yet formed a clear research enthusiasm.

\section{$\underline{h} \underline{\text { aII }} \odot$}

Table 1. Author analysis

\begin{tabular}{|c|c|c|c|c|c|c|c|}
\hline Freq & Burst & Centrality & $\Sigma$ & PageRa. & Keyword & Author & Year \\
\hline 3 & & 0.00 & 1.00 & 0.00 & & Xingron Bao & 2010 \\
\hline 2 & & 0.00 & 1.00 & 0.00 & & Weihua Wang & 2009 \\
\hline 2 & & 0.00 & 1.00 & 0.00 & & Ying Wei & 2009 \\
\hline 2 & & 0.00 & 1.00 & 0.00 & & XiangJian Geng & 2009 \\
\hline 2 & & 0.00 & 1.00 & 0.00 & & Hong Wen & 2018 \\
\hline 2 & & 0.00 & 1.00 & 0.00 & & Dong Zhu & 2011 \\
\hline 2 & & 0.00 & 1.00 & 0.00 & & Taisheng Cai & 2011 \\
\hline 2 & & 0.00 & 1.00 & 0.00 & & Feng Gao & 2007 \\
\hline 2 & & 0.00 & 1.00 & 0.00 & & Xin Peng & 2014 \\
\hline 2 & & 0.00 & 1.00 & 0.00 & & Dianbo Gao & 2009 \\
\hline 2 & & 0.00 & 1.00 & 0.00 & & & \\
\hline
\end{tabular}




\begin{tabular}{|c|c|c|c|c|c|c|c|}
\hline 2 & & 0.00 & 1.00 & 0.00 & & Haiwei Jia & 2012 \\
\hline 2 & & 0.00 & 1.00 & 0.00 & & Yan Zhao & 2012 \\
\hline 2 & & 0.00 & 1.00 & 0.00 & & Xiaoyu Li & 2007 \\
\hline 2 & & 0.00 & 1.00 & 0.00 & & Wenjie Ceng & 2011 \\
\hline 2 & & 0.00 & 1.00 & 0.00 & & Xifeng Ceng & 2008 \\
\hline 2 & & 0.00 & 1.00 & 0.00 & & Jianjun Zheng & 2016 \\
\hline 2 & & 0.00 & 1.00 & 0.00 & & Caorun Lin & 2009 \\
\hline 2 & & 0.00 & 1.00 & 0.00 & & Li Zhou & 2011 \\
\hline
\end{tabular}

Table 2. Institutional analysis

\begin{tabular}{|c|c|c|c|c|c|l|}
\hline Freq & Burst & Centrality & $\Sigma$ & PageRa. & Keywords & \multicolumn{1}{|c|}{ Author } \\
\hline 3 & & 0.00 & 1.00 & 0.00 & & Yunnan Administration College \\
\hline 3 & & 0.00 & 1.00 & 0.00 & & $\begin{array}{l}\text { School of Public Administration. Central South University of } \\
\text { economics and law }\end{array}$ \\
\hline 2 & & 0.00 & 1.00 & 0.00 & & School of Business Administration. Henan University \\
\hline 2 & & 0.00 & 1.00 & 0.00 & & Central South University of economics and law \\
\hline 2 & & 0.00 & 1.00 & 0.00 & & $\begin{array}{l}\text { School of Philosophy and Public Administration. Henan } \\
\text { University }\end{array}$ \\
\hline 2 & & 0.00 & 1.00 & 0.00 & & $\begin{array}{l}\text { College of Educational Sciences. Henan University } \\
\text { Institute of Political Science. Chinese Academy of Social } \\
\text { Sciences }\end{array}$ \\
\hline 2 & & 0.00 & 1.00 & 0.00 & & $\begin{array}{l}\text { School of Public Administration. South China University of } \\
\text { Technology }\end{array}$ \\
\hline
\end{tabular}

\subsubsection{Institutional Analysis}

From the perspective of publishing institution, the institutions that have published papers the most are Yunnan School of Administration, School of Public Administration, Zhongnan University of Economics and Law; followed by School of Business Administration, Henan University, Institute of Political Science, Chinese Academy of Social Sciences, and School of Public Administration, South China University of Technology (Table 2). The regions of publishing institutions are widely distributed in the northern, central, southern and southwestern regions of China. However, the number of publications by these institutions is still relatively small, and the level of institutions is mostly relatively low, indicating that the level of research on the issue of civil servants' job burnout needs to be improved. Double-first-class universities should pay attention to this issue.

\subsubsection{Periodical Distribution}

Among the 163 papers on civil servants' job burnout, only 26 are from Chinese Core Journals (CSSCI), accounting for $15.95 \%$ of all journals. Figure 2 shows the number of papers published in core journals. From the perspective of journal types, there are 15 journals belonging to social sciences, 3 journals belonging to psychology, and 1 journal belonging to statistics. Judging from the time of publication, there were papers on official burnout in China in 2001, while papers in core journals were published only in 2008. In terms of the number of publications, the journal "Chinese Public Administration" has the largest number of publications, with a total of 5 papers, accounting for $19.23 \%$ of all core journal documents. It shows that the degree of specialization of research on official burnout needs to be improved. 


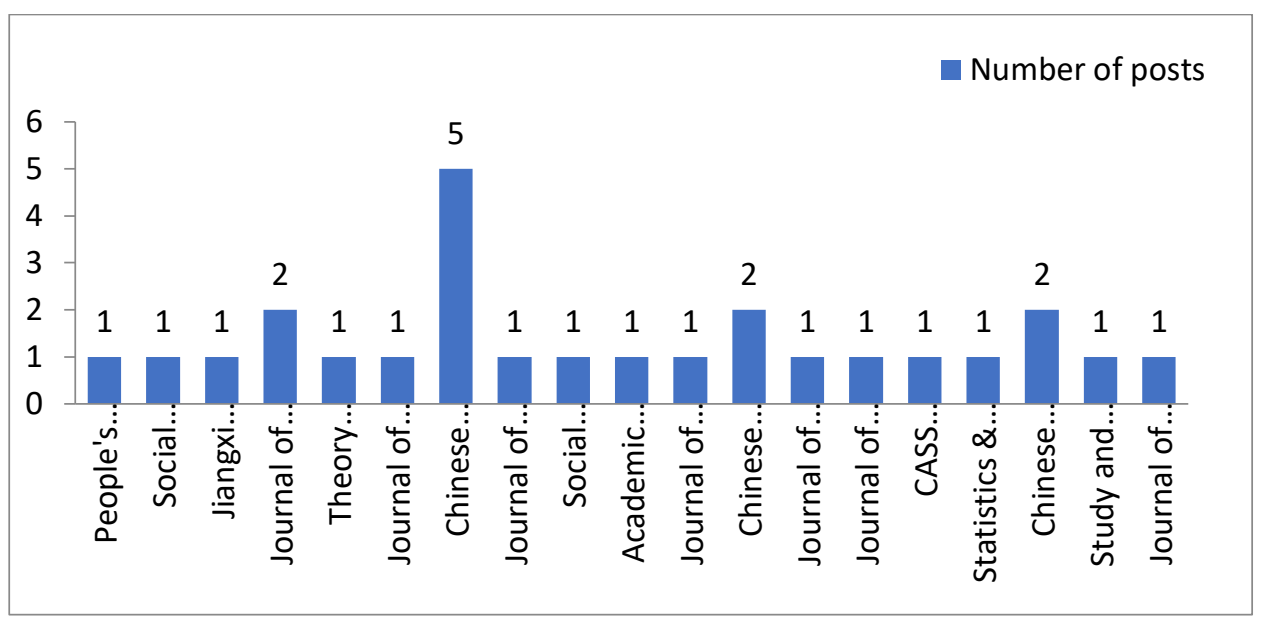

Figure 2. Number of papers published in core journals

\section{CONTENT ANALYSIS OF CIVIL SERVANT BURNOUT RESEARCH}

Keywords reflect the research hotspots and focus of scholars' papers. Analysis of keywords in the documents can quickly find the main content of the author's paper. Therefore, in this study, the CiteSpace software was used to set the Keyword as a node type. The documents on the subject of civil servants' job burnout published in all journals has been visualized and analyzed, and the keyword map of these 163 papers can be obtained.

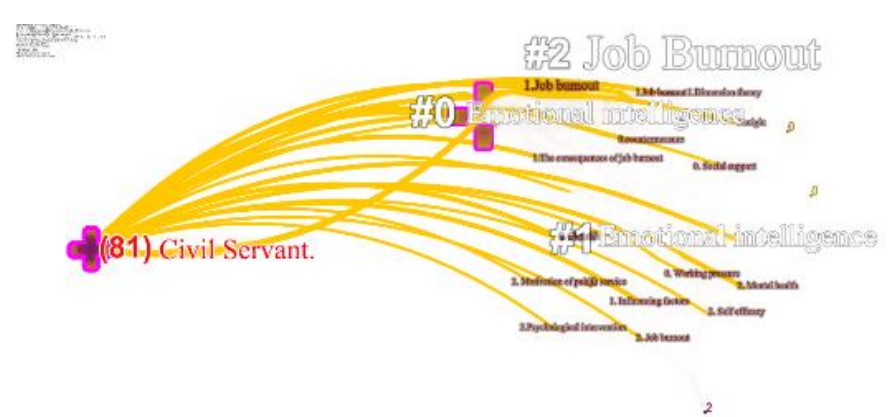

Figure 3. Keyword knowledge map of job burnout research

It can be seen from Figure 3 that the research focus of these documents is on the job burnout of civil servants, and the term emotional intelligence is the most closely related to job burnout. The so-called emotional intelligence means that the manager's ability to understand and control the emotions of himself and his colleagues will determine whether the company can have a better business performance. This term comes from the business field. It was first proposed by Salove (Yale University) and Mayer (New Hampshire University). It refers to "individuals monitor other people's emotion, and identify this information to guide their thoughts and behaviors"[1]. Through the following highfrequency keyword table for burnout research, we can analyze the research hotspots and content of these documents. It can be clearly seen from Table 3 that among these keywords, job burnout is the core keyword, with the highest frequency of 139 times, civil servants 90 times, grassroots civil servants 24 times, countermeasures 8 times, and cause 7 times. The frequency of work stress is 6 times, the frequency of mental health is 5 times, the frequency of self-efficacy is 4 times, and the frequency of administrative workplace ecology is relatively high.

Table 3. Keywords of job burnout research

\begin{tabular}{|c|c|c|c|}
\hline Keywords & Frequency & Keywords & Frequency \\
\hline Burnout & 141 & Personnel Administration & 3 \\
\hline Civil servant & 114 & Psychological capital & 2 \\
\hline Sources of Burnout & 13 & Psychological intervention & 2 \\
\hline Countermeasure analysis & 10 & Officialdom & 2 \\
\hline Work pressure & 6 & Local government & 2 \\
\hline Mental health & 5 & Public service motivation & 2 \\
\hline Self-efficacy & 4 & Government officials & 2 \\
\hline
\end{tabular}




\begin{tabular}{|c|c|c|c|}
\hline Administrative workplace ecology & 4 & Three-dimensional theory & 2 \\
\hline Social support & 3 & ERG theory & 2 \\
\hline Incentives & 3 & Burnout Consequences & 2 \\
\hline
\end{tabular}

\subsection{Contextual Analysis of Research Keywords}

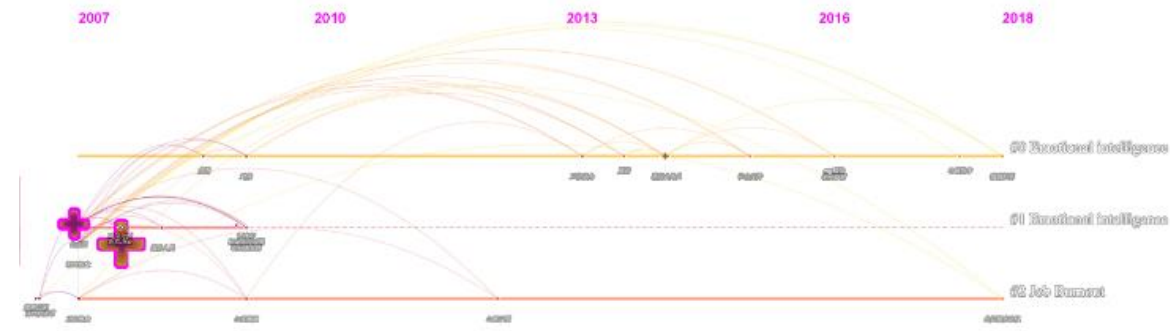

Figure 4. Time Context of Job Burnout Research

Taking the timeline as the axis to analyze, it is possible to get the context of changes in the research keywords related to job burnout of civil servants over time. From Figure 4 we can see that the focus of the study in 2007 was on party and government cadres, self-efficacy, analysis of influencing factors, civil servants and causes of their job burnout; since 2007, the phenomenon of job burnout in the civil service industry has gradually become serious, which has attracted the attention of scholars and all walks of life. This has caused scholars to further analyze the influencing factors and countermeasures of this status.[2]; the hot words studied in 2010 were mental health, job burnout and countermeasures; since 2010, people's mental health problems have become a fact of concern from all walks of life, which affected scholars' research on job burnout and tended to analyze it from the perspective of mental health; the hot words of research in 2013 were psychological intervention and work stress; Since 2013, the working environment, organization evaluation mechanism and other issues of the civil service industry have attracted people's attention, and scholars began to focus on the work pressure of civil servants; the hot words of research in 2016 were social support, government intervention and ERG theory; since 2016, scholars have shifted from the previous personal and organizational levels to analyze the impact of social factors on civil servants' job burnout, and scholars have begun to use various theoretical methods to analyze job burnout in order to find more scientific and objective conclusions; the hot words of research in 2018 were public service motivation, psychological capital and incentive mechanism; After 2018, scholars' research focus turned to the institutional aspect. Scholars began to study from the perspective of incentive mechanism and public service motivation, and looked for breakthrough in institutional direction to solve the problem of job burnout.[3].

\subsection{Keyword Analysis}

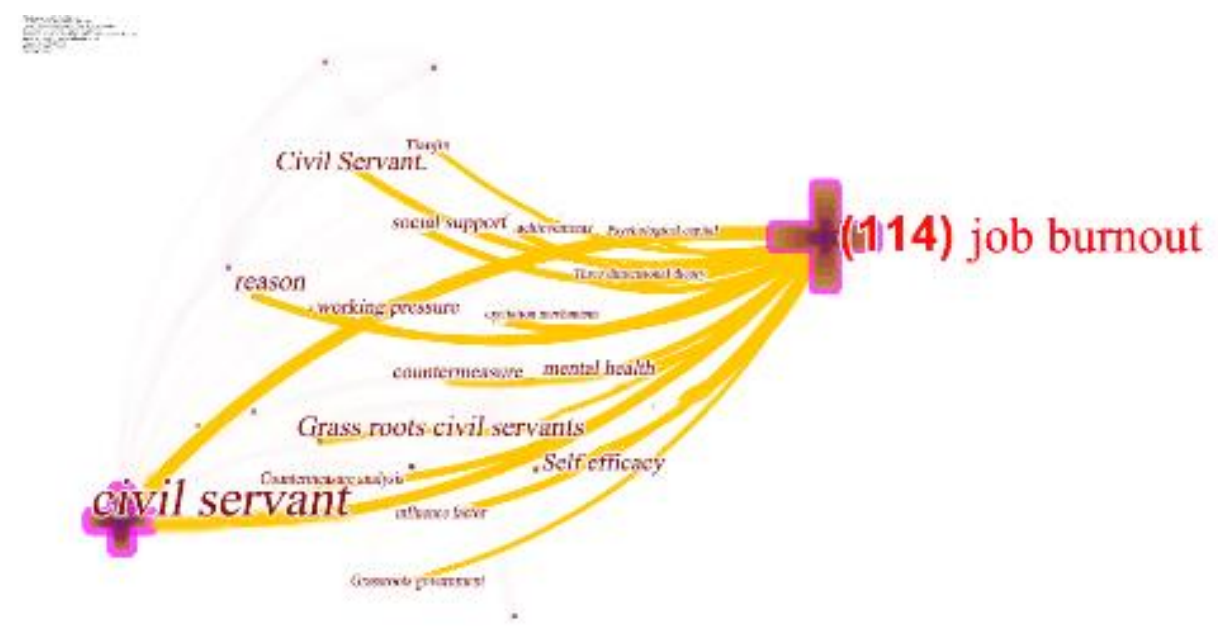

Figure 5. Keyword co-occurrence network of job burnout 


\subsubsection{Burnout}

The keyword co-occurrence network obtained with job burnout as the center is shown in Figure 5. When the scholars study the job burnout, they mostly study the causes, influencing factors and countermeasures of job burnout. There are still many papers that use empirical analysis methods to measure from the three dimensions of job burnout, emotional exhaustion, dehumanization, and personal accomplishment. A sample survey is used to analyze the causes of job burnout in the research subjects. The concept of job burnout was first proposed by Freudenberger in 1974. He believes that job burnout is a symptom of emotional exhaustion that is most likely to occur in the helping industry. Subsequently, then, Maslach and others called the psychological syndrome caused by responding to long-term emotional and interpersonal stressors at work as job burnout. The research shows that job burnout is composed of three dimensions: emotional exhaustion, depersonalization, and diminished personal achievement. It is generally believed that job burnout is an extreme reaction when an individual fails to cope with work pressure smoothly. It is a state of exhaustion of emotions, attitudes, and behaviors that an individual produces under long-term stress experience.

\subsubsection{Public Service Motivation}

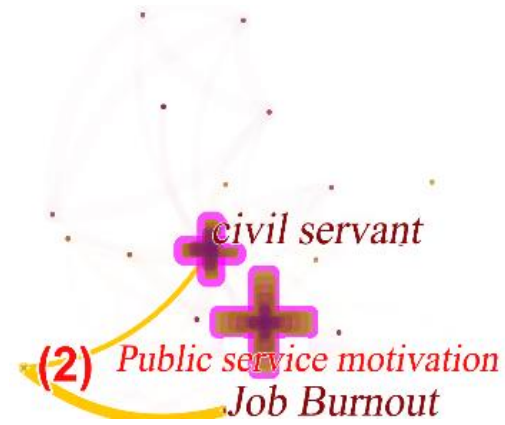

Figure 6. Keyword co-occurrence network of public service motivation

Taking public service motivation as the center, the keyword co-occurrence network obtained is shown in Figure 6 . Public service motivation is the psychological tendency of individuals to respond mainly or entirely based on the motivations of public institutions and organizations. The authors of the documents use interviews and questionnaires, taking public service motivation as the independent variable, and use the three sub-dimensions of job burnout-work mood, work attitude, and sense of work accomplishmentas dependent variables to conduct statistical analysis, and further analyzes the internal relationship between public service motivation and job burnout of civil servants.

\subsection{Analysis of Research Methods and Their Changing Trends}

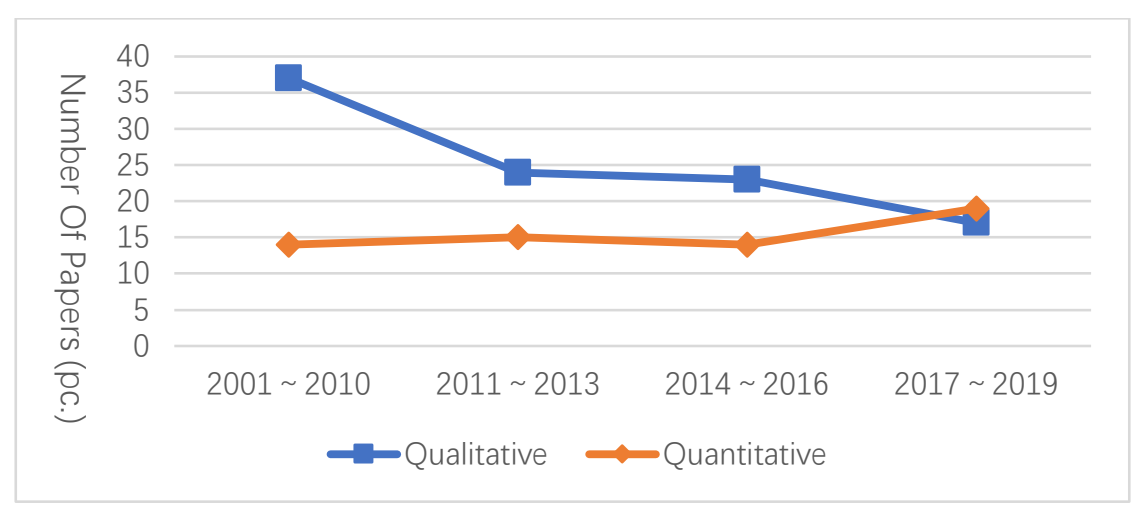

Figure 7. The trend chart of changes in the research methods of civil servants' job burnout from 2001 to 2019

According to the feedback in Figure 7, it can be clearly observed that the number of papers using qualitative research methods from 2001 to 2019 is generally more than that using quantitative research, but the magnitude of the change is large, showing a trend of rapid decline, while the number of papers using quantitative research relatively stable increase; the development process of civil servant job burnout research methods can be roughly divided into three stages; from 2001 to 2013, the number of papers using qualitative research methods declined rapidly, while the number of papers using quantitative research rose steadily; 2013-2016 , both quantitative and qualitative research papers remained stable, but there was a slight decline; from
2016 to 2019, the number of papers published on qualitative research methods once again experienced a decline stage, while the number of papers published for quantitative research accelerated and increased. During this period, it exceeded the number of qualitative research papers for the first time, and there is a rising trend; From the analysis results, the quantitative analysis method of studying civil servants job burnout has become a development trend in the past 18 years, which shows that the method of quantitative analysis of job burnout has its advantages. It is more and more favored by domestic researchers to recognize the advantages of its extensive understanding of society, 
objectivity of research conclusions, reliability of data collection and other advantages.

\subsection{Analysis of the Causes of Civil Servants' job Burnout}

Maslach's three-dimensional burnout theory classifies the main manifestations of job burnout as: (1) Emotional Exhaustion, it means that the individual's emotions are in a state of extreme fatigue, emotional resources are exhausted, and the job enthusiasm is completely lost; (2) Depersonalization, which is the individual treats others around him with a negative and insensitive attitude, lacks sympathy for people, and even treats others as inanimate; (3) Reduced personal accomplishment means that individuals' appraisal of the meaning and value of their own work decreases, their sense of self-efficacy is lost, and they often feel incompetent for the work they are engaged in, and they cannot feel the achievement in the work[4]

By categorizing the causes of civil servants' job burnout in the documents, it is found that most documents based on the three-dimensional theory of Marler's poems divide the causes of civil servants' job burnout into three aspects: individual, organization, and the society. Individual aspects can be further divided into three main reasons: psychological problems, personality differences, and personal abilities; organizational aspects are mainly composed of two parts: one is organizational style--an organization's internal management and communication is not smooth and lacks inclusive leadership style; the second is evaluation mechanism is difficult to promote, failure of the evaluation mechanism, insignificant incentive effects, etc.; at the social level, the public's expectations and the public environment will also have an impact on the job burnout of civil servants: generally speaking, the public has over-expectations for civil servants. The resulting social supervision will increase the work pressure of civil servants. In addition, the public environment is changing with each passing day and the changes in macroeconomic policies are accelerating, making it impossible for civil servants to adjust their policies in time, causing psychological pressure invisibly [5].

\section{RESEARCH CONCLUSION AND PROSPECTS}

\subsection{Research Conclusion}

This paper uses Citespace bibliometric software to analyze 163 relevant documents on civil servant burnout in all journals in the China Academic Journals Network Publishing Library (CNKI) from 2001 to 2019, and draws the following conclusions:

From the disciplinary perspective of research hotspots, we can get the changing context of the research keywords about the job burnout of civil servants with the change of time. It closely focuses on the main individual psychological problems such as self-efficacy, mental health, work pressure incentive mechanism, and lacks the overall research of human resource management. Although the study of psychology is the foundation of human resource management and the use of the perspective of psychology helps to expand the understanding of civil servants' burnout, but civil servants' burnout is essentially a human management problem, and it will be even more critical and profound based on the perspective of human resource management [6].

From the perspective of the number of papers in journals, the total number of research papers on civil servant burnout published in China is relatively small in the past 18 years, indicating that the attention and degree of research on official burnout are obviously insufficient [7]. The degree of specialization of the research on official burnout in China needs to be improved. Moreover, the research on job burnout of civil servants is fluctuating and has not yet formed a clear research enthusiasm.

From the perspective of the authors and the posting institutions, the regions of the posting institutions are widely distributed in the northern, central, southern, and southwestern of China. However, the number of papers published by these institutions is relatively small, and the institutions' level is mostly relatively low. Scholars who are engaged in the research on job burnout of civil servants have a relatively shallow level of research on burnout, and the scope and depth of research are insufficient. Although some core research teams have been formed in the field of civil servant burnout, the connection between the teams is weak, and most scholars are still in independent research. There are certain academic connections between research institutions, but the intensity of cooperation is low, especially in the lack of institutional cooperation between different regions [8].

\subsection{Research Prospects}

Firstly, scholars should expand the research on civil servant burnout based on the existing research results in this field and try to explore from multiple perspectives and fields in future research. The research on civil servant burnout still needs starting from the background of the discipline of resource management, and systematically examines the influence of management in the field of social organizations on "human" and deepens and perfects the measurement [9] Secondly, we should pay more attention to the analysis and discussion of civil servants' job burnout at the research level. As a social public officer, civil servants play a vital role in exporting public products and providing public services, they are the core force to maintain the operation of society. Therefore, strengthening the research on civil servants' job burnout can get the society's attention to the physical and mental health of civil servants, which will help promote social stability [10].

Finally, in order to improve the quality and social influence of civil servant burnout research in China, it is urgent to promote cross-regional scientific research cooperation. The specific path is to promote the construction of the localized 
theory of China's unique civil servant job burnout phenomenon. The logic behind it is the description and explanation of China's unique human resource management phenomenon. In different regions, researchers should establish a common research field, enhance the dialogue function with each other, and reduce regional differences. There are still some shortcomings in this study. Constrained by the limitation of the sample size of the document, the analysis results of this paper are not sufficient, but this can precisely reflect the deficiencies in the research field of civil servants' job burnout. Referencing foreign studies on official burnout, hoping to make further suggestions and references for the research on civil servants' burnout in China, and it will be the direction that researchers need to continue to work hard on.

\section{REFERENCES}

[1] Wang Liu. Exploring the job burnout of grassroots civil servants from organizational factors $[\mathrm{J}]$. Think Tank Era, 2018-07-30. (In Chinese)

[2] Zhijie Qin, Zhi Qu, Dingcai Zhou. Research on job burnout of grassroots civil servants based on motivation theory[J]. The Forum Leadership Science, 2018-10-16. (In Chinese)

[3] Yan Li, Chunku Zhu i. Research on the Influencing Factors and Intervention Strategies of Selected Students' Job Burnout from the Perspective of Psychological Contract[J]. Tribune of Study, 2019-6-15. (In Chinese)
[4] Quanfeng Shu, Yachan Liu. Public Service Motivation, Leadership and Job Burnout of Grassroots Poverty Alleviation Cadres[J]. Social Sciences of Beijing, 2019-7-16. (In Chinese)

[5] Jiahui Lv, Analysis of Job Burnout of Civil Servants in Villages and Towns-Based on the Perspective of ERG Theory [J] Business, 2016-7-13. (In Chinese)

[6] Lianlin Jiang. Analysis of the job burnout of civil servants in my country[J]. Human Resources Management, 2014-8-8. (In Chinese)

[7] Wei Zhou. Research on the causes of civil servants' job burnout and corresponding countermeasures[J]. Research of Administration Science, 2019-5-15. (In Chinese)

[8] Weixia Lu., Xiaoming Xu, Chaojie Wang. Research on Evaluation Indexes and Influencing Factors of Grassroots Civil Servants' Job Burnout [N]. Journal of Northeastern University, 2018-9-12. (In Chinese)

[9] Shiyang Liao, Yu Zhao. Thoughts on improving self-efficacy of civil servants[J]. Leadership Science, 2017-7-4. (In Chinese)

[10] Fulin Fan. An Empirical Study on the Psychological Capital and Job Burnout of Grassroots Leaders [N]. Journal of Liaoning Academy of Governance, 2018-3-20. (In Chinese) 